M. Guarascio et al (eds.), Advanced Geostatistics in the Mining Industry, 391-399. All Rights Reserved. Copyright (C) 1976 by D. Reidel Publishing Company, Dordrecht-Holland.

\title{
SOME PRACTICAL COMPUTATIONAL ASPECTS OF MINE PLANNING
}

Isobel Clark,

Dept. of Mining and Mineral Technology, Imperial College,

Prince Consort Road, London, SW7, England.

\section{INTRODUCTION}

During the planning stage of a new mine, a company often requires ore reserve estimates on a "mining block" scale, as well as large scale or global estimates. The ideal report contains plans on a bench by bench basis, showing the average grade of each block with perhaps an associated confidence limit.

Many papers published to date (cf. Journel and Huijbregts 1973) dealing with three dimensional estimations using borehole data, have produced estimates in two stages: firstly, "mineable thickness" is established for each borehole in turn, together with an average grade; secondly, global and other estimates are made in two dimensions, treating the boreholes as "points" on a surface. This is very useful for planning optimal open-pit outlines (cf. Journel and Sans, 1974), and for locating generally high and low grade areas. However, for actual dayto-day or Month-to-month mine planning it is often necess ary to produce estimates on a block by block and bench by bench basis.

The estimation of blocks in three dimensions from borehole data can be very simple, if: all boreholes are drilled on a regular grid; are of roughly the same length - at least in the area of concern - and are continuous over the lengths which have been assayed. However, problems of several kinds may be encountered in practice: the boreholes may not have been drilled on a grid - or, if they were, all the grid points may not have been (or may never be) included; the boreholes and/or their intersections with the ore body may be of widely differing lengths; there may be gaps in the cores which have not been assayed - for one reason or another; core sections may have been valued in different lengths; there may be differential core recovery within different areas of the deposit or at different points down the same borehole.

\section{KRIGING}

For the purposes of Kriging, it is necessary to calculate the interrelationships (in terms of the semi-variogram) between the samples used in the estimation process and the unknown area, and also between all possible combinations of pairs within the sample set, This is usually expedited by the use of standard Auxiliary Functions, which define the value of the semivariogram for a small number of standardised geometrical situations, Since the auxiliary functions in three dimensions are sometimes difficult (especially for spherical schemes) and tedious to evaluate - 
consuming a lot of subjective work time practical computational evaluation in three dimensions usually takes one of two forms.

The standard auxiliary functions can be evaluated by setting up numerical approximations to the integrals involved. For instance, the auxiliary function for the average semivariogram between a block measuring | by b by h units and a point on one corner of that block would be defined as:

$$
H(\ell, b, h)=\frac{1}{\ell b h} \int_{0}^{h} \int_{0}^{b} \int_{0}^{\ell} r\left(\sqrt{x^{2}+y^{2}+z^{2}}\right) d x d y d z
$$

This would be evaluated numerically using, say, Simpson's rule or some such standard method.

Alternatively, the integrals could be substituted by summations over a very large number of points and calculated as:

$$
\begin{aligned}
& H^{\prime}(l, b, h)=\frac{1}{\left(n_{1}+1\right)\left(n_{2}+1\right)\left(n_{3}+1\right)} \sum_{i=0}^{n_{1}} \sum_{j=0}^{n_{2}} \sum_{k=0}^{n_{3}} \gamma\left(d_{i j k}\right) \\
& \text { where } d_{i j k}^{2}=\left(i \frac{h}{n_{1}}\right)^{2}+\left(j \frac{b}{n_{2}}\right)^{2}+\left(k \frac{l}{n_{3}}\right)^{2}
\end{aligned}
$$

The kriging procedure could then be carried out as normal provided that the standard auxiliary functions were adequate for the situation.

The other method is to forget the auxiliary functions and to represent each sample or block by a series of points. For example, a block may be represented by a network of points throughout the volume. A borehole used in estimating the block could be approximated by a onedimensional series of points very close together. The average semi-variogram between core and block would then be approximated by the average semi-variogram for all pairs of the type (point on core, point in block\}. As an illustration in two dimensions, the configuration in Figure la could be approximated by the sets of points shown in Figure lb.
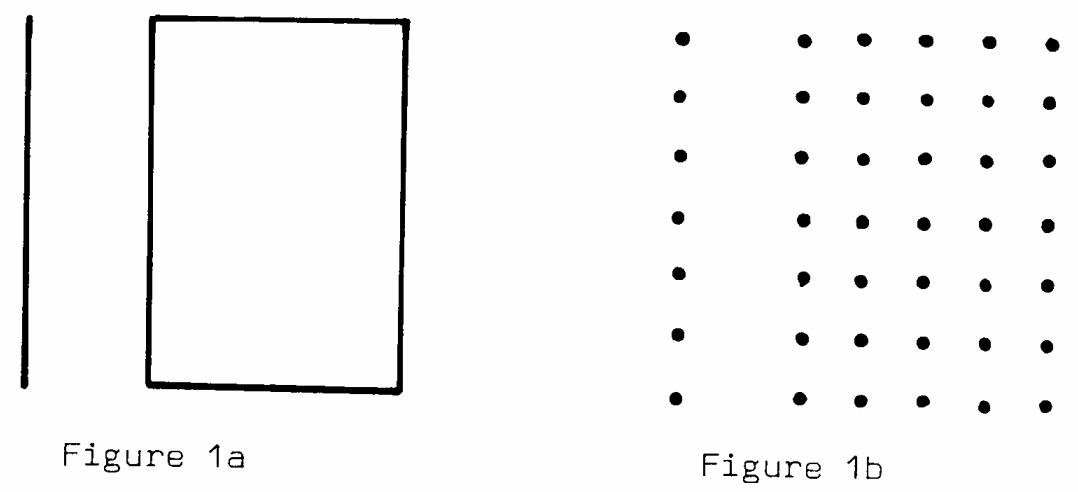

This approach avoids the problem of having to reduce all the practical geometric situations to the ideal ones. For instance, for evaluating a core to block relationship, the configuration shown in Figure 2 is the only one covered by an auxiliary function. 


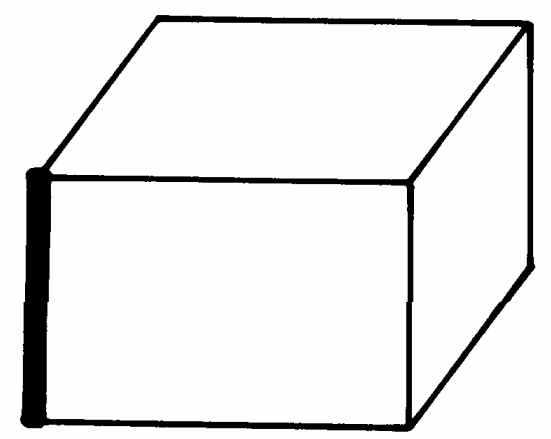

Figure 2

However, this allows only for cores of the same length as one side of the block, and parallel to that side. This means that only the borehole intersection with each 'bench' could be used to estimate the block if the standard auxiliary functions were

If the bench width is much smaller than the range of influence, this is obviously unrealistic. Whilst the second numerical approach avoids the problems mentioned, it does involve a lot of computer time and a fair amount of approximation error.

\section{THE FUNCTION}

The introduction of a new auxiliary function would seem to ease some of the necessary approximations inherent in both of the aforementioned procedures. A new function is proposed to cope with the situation illustrated in Figure 3. That is, the average semi-variogram between two parallel segments of lengths $\mid$ and $b$, a distance $h$ apart, whose ends are offset by a distance d.

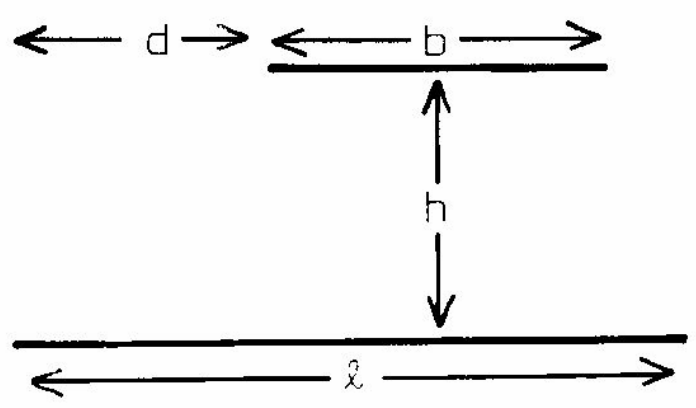

\section{Figure 3}

In practice, it has been found advantageous to define the function so that $b$ is always less than I, $\mathrm{h}$ is always positive, and d may take any value - positive or negative. To distinguish this 
function from the auxiliary function $\gamma(\mathrm{h} ; \mathrm{l})$ for two parallel segments of length $\mathrm{t}$ a distance $\mathrm{h}$ apart, it has been designated as $\boldsymbol{\mathcal { \lambda }}(\mathrm{l}, \mathrm{b}, \mathrm{h}, \mathrm{d}) . \boldsymbol{\mathcal { \lambda }}$ is the Hebrew letter Gimel. The definition is:

$$
\boldsymbol{\alpha}(l, b, h, d)=\frac{1}{l b} \int_{0}^{l} \int_{d}^{d+b} \gamma\left(\sqrt{(x-y)^{2}} \bar{h}^{2}\right) d x d y
$$

\section{USES OF THE FUNCTION}

The function will cope easily with different lengths of borehole cores, and with differential recovery rates, since it will compare any length of core with any other. Cores along the same borehole can also be handled simply by setting h equal to zero.

The problem of representing blocks still remains. It is suggested that instead of a network of points distributed evenly within the block, a suitable representation for the block would be as a "matrix" of segments perpendicular to the plane of the bench. That is, looking down on the block, one would view a grid of points, and from any side one would see a set of vertical segments distributed evenly along the side.

The block with block relationship would then be approximated by averaging all possible pairs of the segments within the block - these relationships being simply evaluated by the auxiliary function $\gamma(\mathrm{h} ; \mid)$. Core with block relationships can also be evaluated by using $\boldsymbol{\lambda}(l, \mathrm{~b}, \mathrm{~h}, \mathrm{~d})$ to furnish the semi-variogram between the core length and each of the segments within the block in turn. This allows relatively rapid calculation of core with block semi-variograms for any relative positions of core and block. It also allows the use of core lengths above and below the bench containing the block to be included in the estimation procedure,

\section{THE SPHERICAL MODEL}

It is the authors experience that most mineral deposits particularly those of low concentration minerals, cassiterite, nickel, copper, uranium - follow the Spherical or Matheron model of the semi-variogram, or mixtures of this model. Since this is also the only model that poses any real problems in the production of auxiliary functions, the remainder of this paper is couched in terms of this model.

The function $\mathcal{2}$ was produced for the spherical scheme, but the necessity for brevity in this paper prevents the publication here of either the numerous formulae or the FORTRAN IV FUNCTION segment written by the author. However, copies of these may be obtained from the author on personal request. 
Various investigative work was then necessary to determine an acceptable level of approximation for the block relationships - i.e. an acceptable level of density for the segments representing the block. It seemed intuitively sound to assume that the approximation of a two dimensional panel by a grid of points would be equivalent to representing a three dimensional block by a grid of segments. Therefore, a considerable amount of work was done on the simpler approximation. For illustration, the auxiliary function $F(l, b)$ for a panel with itself was chosen. This can be evaluated accurately with ease (cf. Clark 1975), so that the errors involved in grid approximation can be calculated precisely.

Two different grids were tested for accuracy, and are shown in Figures $4 \mathrm{a}$ and $4 \mathrm{~b}$.

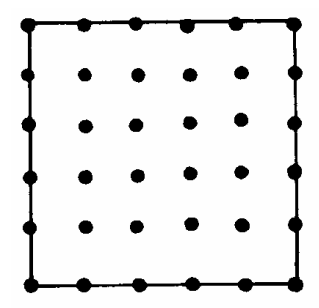

Figure $4 a$

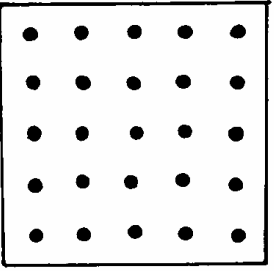

Figure 4b

It was quickly discovered that the latter grid produces remarkably more accurate approximation than $4 \mathrm{a}$. It is obvious that $4 \mathrm{a}$ will, to some extent, overestimate the function, and $4 \mathrm{~b}$ will underestimate it, To illustrate the difference in accuracy Table 1 shows the percentage error incurred in the two approximations for equivalent grid spacings. That is, the first line of the table, giving grid space 5, means that a five by five grid of points was used in Figure 4b. To achieve the same spacing in Figure 4a, a six by six grid is necessary, and so on for each entry. The panel size used in this example was la by $1 \mathrm{a}$, where a is the range of influence of the model.

Since the second type of grid is obviously far superior, it remains to choose a level of acceptable approximation. It can be seen from Table I that an 18 by 18 grid give four times the accuracy of a ten by ten grid. However, it takes almost eleven times as much computation time. It seemed that a one half per cent error was probably acceptable in practical situations, especially as the computation time for a ten by ten grid was within reasonable bounds, Table 2 shows the percentage error for various panel sizes using such a grid. The Table shows that the error remains fairly consistent over widely varying panel sizes.

A brief study was also undertaken to illustrate situations in which it may be desirable to use cores above and below the bench containing a block under consideration. The extension variance of a central borehole to a block was calculated for various lengths of core. This example could obviously be repeated for an infinite number of combinations of size of block and range of influence. However, a simple example, encountered by the author on a disseminated nickel deposit in northern Norway was chosen. Here, there was a range of influence of fifty metres, a bench width of ten metres and a block size of 25 by 25 metres. 
Taking a length of core centred within the block and varying that length from two metres to fifty, the results in Table 3 were produced. For comparison, the same evaluations were made for a 10 by 10 metre block.

The Table shows that for a $25 \mathrm{~m}$ by $25 \mathrm{~m}$ block the optimal estimation is made from a $30 \mathrm{~m}$ core - that is, the core intersection with not only the bench containing the block, but also with the bench above and the bench below should be considered However, for a $10 \mathrm{~m}$ by $10 \mathrm{~m}$ block, a core length of about $16 \mathrm{~m}$ is optimal .

This kind of study is very valuable for aiding decisions on the amount of data to be included in the kriging procedure to produce truly optimal estimators.

\section{CONCLUSION}

The uses of the function $\boldsymbol{\lambda}$ in practical mine planning a obviously many and varied. They are not restricted to three dimensional applications, since the function itself is actual twodimensional. One disadvantage of the function as defined is that it will deal only with approximately vertical boreholes, since the segments must be parallel. However, it is hoped to produce a truly three dimensional version of $\mathcal{\alpha}$ in the near future.

\section{ACKNOWLEDGEMENTS}

The author wishes to thank the Norwegian Geological Survey under whose auspices the derivation of the function $\boldsymbol{\lambda}$ was initiated and Mr A.A. Heidecker of the Mining Dept. at Imperial for introducing me to the Hebrew alphabet. The final form was completed at Imperial College, and implemented on the CDC 6400 installation

\section{REFERENCES}

Clark, I 1975 "Some Auxiliary Functions for the Spherical Model of Geostatistics", Computers \& Geosciences Vol 1 No 4.

Journel, A.G. \& Huijbregts, Ch. 1973 "Estimation of Lateritic Type Orebodies" International Symp. on Computers in Mineral Industry, S.A.I.M.M., Jo'Burg.

Journel, A.G. \& Sans, H 1974 "Ore-grade control in sub-horizontal deposits" Trans. Inst. Min. Metall. Vol 83. 
Table 1: Percentage Approximation Error

\begin{tabular}{|c|c|c|}
\hline Grid spacing & Grid type a & Grid type b \\
\hline 5 & -16.28 & 2.1151 \\
6 & -13.85 & 1.4204 \\
7 & -12.05 & 1.0185 \\
8 & -10.66 & 0.7656 \\
9 & -9.55 & 0.5962 \\
10 & -8.66 & 0.4774 \\
11 & -7.92 & 0.3908 \\
12 & -7.29 & 0.3257 \\
13 & -6.76 & 0.2757 \\
14 & -6.29 & 0.2363 \\
15 & -5.89 & 0.2048 \\
16 & -5.54 & 1,792 \\
17 & -5.22 & 0.1581 \\
18 & -4.94 & 0.1406 \\
\hline
\end{tabular}

Table 2: Percentage Approximation Error for Panels

\begin{tabular}{|c|cccccc|}
\cline { 2 - 7 } \multicolumn{1}{c|}{} & \multicolumn{6}{|c|}{$1 / \mathrm{a}$} \\
\hline $\mathrm{b} / \mathrm{a}$ & 0.1 & 0.2 & 0.3 & 0.4 & 0.5 & 1.0 \\
\hline 0.1 & 0.520 & 0.525 & 0.535 & 0.543 & 0.549 & 0.535 \\
0.2 & 0.525 & 0.515 & 0.513 & 0.511 & 0.508 & 0.458 \\
0.3 & 0.535 & 0.513 & 0.506 & 0.501 & 0.494 & 0.429 \\
0.4 & 0.543 & 0.511 & 0.501 & 0.494 & 0.486 & 0.413 \\
0.5 & 0.549 & 0.508 & 0.494 & 0.486 & 0.477 & 0.401 \\
& & & & & & \\
0.6 & 0.553 & 0.504 & 0.487 & 0.476 & 0.467 & 0.390 \\
0.7 & 0.553 & 0.497 & 0.476 & 0.465 & 0.455 & 0.378 \\
0.8 & 0.550 & 0.487 & 0.464 & 0.450 & 0.440 & 0.364 \\
0.9 & 0.544 & 0.474 & 0.448 & 0.433 & 0.422 & 0.349 \\
1.0 & 0.535 & 0.458 & 0.429 & 0.413 & 0.401 & 0.332 \\
& & & & & & \\
1.1 & 0.526 & 0.443 & 0.412 & 0.394 & 0.382 & 0.316 \\
1.2 & 0.521 & 0.433 & 0.397 & 0.379 & 0.366 & 0.303 \\
1.3 & 0.528 & 0.432 & 0.393 & 0.371 & 0.357 & 0.293 \\
1.4 & 0.527 & 0.426 & 0.384 & 0.362 & 0.347 & 0.284 \\
1.5 & 0.543 & 0.434 & 0.387 & 0.361 & 0.343 & 0.279 \\
\hline
\end{tabular}


Table 3: Extension variance of a Central Core to a Block for sill equal to 1.0

\begin{tabular}{|c|c|c|}
\hline Core length & $10 \mathrm{~m}$ by $10 \mathrm{~m}$ & $25 \mathrm{~m}$ by $25 \mathrm{~m}$ \\
\hline 2 & 0.0716 & 0.1760 \\
4 & 0.0563 & 0.1589 \\
6 & 0.0440 & 0.1436 \\
8 & 0.0347 & 0.1302 \\
10 & 0.0282 & 0.1186 \\
12 & 0.0241 & 0.1086 \\
14 & 0.0221 & 0.1002 \\
16 & 0.0219 & 0.0932 \\
18 & 0.0231 & 0.0875 \\
20 & 0.0255 & 0.0829 \\
22 & 0.0289 & 0.0795 \\
24 & 0.0332 & 0.0770 \\
26 & 0.0382 & 0.0755 \\
28 & 0.0438 & 0.0748 \\
30 & 0.0500 & 0.0749 \\
32 & 0.0568 & 0.0757 \\
34 & 0.0639 & 0.0772 \\
36 & 0.0716 & 0.0793 \\
98 & 0.0796 & 0.0821 \\
40 & 0.0879 & 0.0854 \\
42 & 0.0967 & 0.0892 \\
44 & 0.1057 & 0.0936 \\
46 & 0.1151 & 0.0985 \\
48 & 0.1248 & 0.1038 \\
50 & 0.1348 & 0.1096 \\
\hline
\end{tabular}

